\title{
Extended Resection for Thyroid Disease has Less Operative Morbidity than Limited Resection
}

\author{
Christian A. Seiler, MD, MHA, ${ }^{1}$ Stephan A. Vorburger, MD, MCR, ${ }^{1}$ Ulrich Bürgi, MD, ${ }^{2}$ \\ Daniel Candinas, MD, ${ }^{1}$ Stefan W. Schmid, MD ${ }^{1}$ \\ ${ }^{1}$ Department of Visceral and Transplantation Surgery, Inselspital, University of Bern, 3010 Bern, Switzerland \\ ${ }^{2}$ Department of General Internal Medicine, Inselspital, University of Bern, 3010 Bern, Switzerland
}

\begin{abstract}
Background: Theodor Kocher, surgeon and Nobel laureate, has influenced thyroid surgery all over the world: his treatment for multinodular goiter-subtotal thyroidectomy-has been the "Gold Standard" for more than a century. However, based on a new understanding of molecular growth mechanisms in goitrogenesis, we set out to evaluate if a more extended resection yields better results.

Methods: Four thousand three hundred and ninety-four thyroid gland operations with 5,785 "nerves at risk" were prospectively analyzed between 1972 and 2002. From 1972 to 1990, the limited Kocher resections were performed, and from 1991 to 2002 a more radical resection involving at least a hemithyroidectomy was performed.

Results: The incidence of postoperative nerve palsy was $3.6 \%$ in the first study period and $0.9 \%$ in the second $(P<0.001$, Fisher's exact). Postoperative hypoparathyroidism decreased from $3.2 \%$ in the first period to $0.64 \%$ in the second $(P<0.01)$. The rate of reoperation for recurrent disease was $11.1 \%$ from 1972 to 1990 and $8.5 \%$ from 1991 to $2002(P<0.01)$.

Conclusions: Extended resection for multinodular goiter not only significantly reduced morbidity, but also decreased the incidence of operations for recurrent disease. Our findings in a large cohort corroborate the suggestions that Kocher's approach should be replaced by a more radical resection, which actually was his original intention more than 130 years ago.
\end{abstract}

I 1883, Theodor Kocher, then chairman of the Department of Surgery at the University of Bern, warned about the perils of radical thyroid surgery in a paper entitled: "On extirpation of the goiter and its sequelae". ${ }^{1-6}$ In this publication, based on over 100 very accurately documented cases, he recommended only performing subtotal resections in order to avoid hypothyroidism, recurrent laryngeal nerve palsy, and hypoparathyroidism (which in those days was not very well known or understood). ${ }^{1}$ Based on this experience, sub-

Correspondence to: Christian A. Seiler, MD, e-mail: christian.a.seiler@ insel.ch total thyroidectomies or nodal enucleations became the "criterion standard" of goiter surgery and were performed all over the world over the next 100 years. ${ }^{7}$ In Kocher's day, Switzerland was faced with a staggering goiter epidemic: an impressive $35 \%$ of the children in first grade and $79 \%$ of the children in ninth grade suffered from significant goiter disease. ${ }^{8-11}$ Kocher's innovative understanding of this epidemiologic problem led him to the hypothesis that iodine deficiency was the etiology of goiter disease. He, therefore, advocated the addition of iodine to table salt in Switzerland, which was begun under governmental control in 1922. The result of this ingenious initiative-for which Kocher was awarded the Nobel 
prize-was an impressive drop in the incidence of goiter disease in this country. Thus, iodine deficiency has not been prevalent in Switzerland for over 80 years, ${ }^{12}$ unlike other European countries that have iodine-deficient regions. It offers, therefore, a "laboratory-like" ground for clinical and epidemiologic research.

However, reviewing data on thyroid surgery prospectively collected from 1972 to 1990 , we realized that still a large number of goiters needed surgical treatment and that the incidence of postoperative recurrent thyroid disease is high (11\%). Morbidity associated with this surgery is not negligible: of the referred patients with recurrent disease the preoperative permanent recurrent laryngeal nerve palsy rate $(19.2 \%)$ was forbiddingly high. At our institution the recurrent laryngeal nerve palsy rate was $3.6 \%$ at the initial operation and $5.2 \%$ at reoperation, and the incidence of postoperative hypoparathyroidism was $3.2 \%$. This, and improved surgical infrastructure compared with Kocher's days is why we performed a more extensive approach to thyroid disease after 1990, which demanded the visualization of the recurrent laryngeal nerve and the parathyroid glands. In the present study we wanted to evaluate whether the more extensive goiter resection resulted in more surgery-associated morbidity and/or a decrease in goiter recurrence.

We reviewed our prospectively collected data on all thyroid gland surgeries at our institution from 1972 to 2002 and compared the period from 1972 to 1990, when we were operating according to the Kocher concept of thyroid surgery, with the period from 1991 to 2002, when-based on a new understanding of goitrogenesis-a more extended surgical approach was used. The primary goal of this study was to determine whether the more extensive surgical approach improves the shortand long-term outcome of thyroidectomy and can safely be recommended.

\section{PATIENTS AND METHODS}

This study is a single institutional non-concurrent cohort study of 4,349 goiter operations (corresponding to 5,785 "nerves at risk") performed at the University Hospital of Bern, Switzerland. All patients undergoing a goiter operation at this institution between 1972 and 2002 were included in the study and prospectively followed for the recurrence of laryngeal nerve lesions by direct laryngoscopy, which was performed in all patients before and after surgery and followed over 1 year by independent ENT specialists (loss to follow-up was $1 \%$ ). Since in the present study operative morbidity was the primary out-
Table 1.

Indication for thyroid surgery over the entire observation study period

\begin{tabular}{lcc}
\hline & Number & Percentage \\
\hline Initial operation & 3,904 & \\
Benign nodular goiter & 2,568 & 66 \\
Hyperthyroidism & 796 & 20 \\
Malignancies & 540 & 14 \\
Operation for recurrence & 445 & \\
Benign nodular goiter & 349 & 78 \\
Hyperthyroidism & 22 & 5 \\
Malignancies & 74 & 17 \\
Total operations & 4,349 & \\
Total nerves at risk & 5,785 & \\
\hline
\end{tabular}

come, we included all (benign and malignant) pathologies that led to thyroid surgery. Over the whole study period, patient and operative data were collected prospectively and analyzed retrospectively for transitory and permanent recurrent laryngeal nerve palsy, hypoparathyroidism, and recurrence of goiter disease. Preoperative evaluation during the whole study period included history, clinical evaluation, scintigraphy and/or sonography, FNA in most of the cases, and thyroid hormone status. The indications for the initial operation are listed in Table 1. The indication for surgery was benign nodular goiter in $66 \%$, hyperthyroidism in $20 \%$, and malignancy in $14 \%$. Of the 4,349 operations, 445 were performed for recurrent goiter disease. In $78 \%$ of these 445 patients, the indication for reoperation was recurrent benign, euthyroitic, nodular goiter disease, associated, in particular, with compressive symptoms, rapid thyroid growth, or concern due to malignant alteration. The other $22 \%$ of recurrences were due to malignancy (16\%) and hyperthyroidism (6\%). Patients undergoing operation had to be euthyroid on the day of surgery. Table 2 shows the yearly rate of operations of the patients in the 2 study periods stratified in key indications. The basic demographic data did not differ between the groups (data not shown). In addition, no difference between the 2 cohorts could be detected for demographic data relevant to thyroid disease-the yearly rate of thyroid surgery and the proportion of the various indications for thyroid surgery. Referral patterns remained the same over the study period. Interventions for benign nodular goiter remained between $66 \%$ and $63 \%$ over the whole study period. The rate of hyperthyroidism leading to thyroid surgery was $21 \%$ in the first study period and $20 \%$ in the second. Further subgroup analysis for hyperthyroidism showed a median rate in the 2 study periods of 10 patients and 8 patients operated on for Graves' disease per year (pat/y) $(P=$ not significant 
Table 2.

The 2 observation periods are similar with regard to the frequency of operations and the etiology of the underlying diseases

\begin{tabular}{|c|c|c|c|c|c|}
\hline & \multicolumn{4}{|c|}{ Operations/year } & $\begin{array}{l}\text { Intergroup difference } \\
\text { Significance ( } P \text { value) }\end{array}$ \\
\hline Operations total $(n=4,349)$ & $161(100-174)$ & & $130(114-137)$ & & NS \\
\hline Nerves at risk $(n=5,785)$ & $195(132-217)$ & & $181(180-206)$ & & NS \\
\hline Primary operations $(n=3,904)$ & $143(92-57)$ & 89 & $118(109-124)$ & 92 & NS \\
\hline Hyperthyroidism $(n=796)$ & $25(19-34)$ & 21 & $23(16-31)$ & 20 & NS \\
\hline Malignant goiter $(n=540)$ & $17(13-20)$ & 13 & $22(11-23)$ & 16 & NS \\
\hline Operations for recurrence $(n=445)$ & $17(10-20)$ & 11 & $10(8-13)$ & 8 & 0.002 \\
\hline Benign nodular goiter $(n=349)$ & $13(8-16)$ & 76 & $8(6-10)$ & 79 & NS \\
\hline Hyperthyroidism $(n=22)$ & $1(0-1)$ & 6 & $1(0-1)$ & 6 & NS \\
\hline Malignant goiter $(n=74)$ & $3(1-3)$ & 18 & $1(1-3)$ & 15 & NS \\
\hline
\end{tabular}

NS: Statistically not significant.

The table compares the median operations performed per year in each time period. Values in parentheses represent the lower and upper limits of the $95 \%$ confidence interval. Percentages given refer to the total of each category. Hyperthyroidism and malignant goiter are sub-categorized in the text. $P$ values were calculated using the Mann-Whitney $U$ test and significance levels were set at $P<0.05$.

[NS]). Toxic adenoma (8 vs. 3 pat/y in each period $[P=0.02])$ and toxic nodular goiter (8 vs. 9 pat $/ y$ $[P=\mathrm{NS}])$ were the 2 other reasons for hyperthyroidism leading to operations. The subgroups of malignant goiter disease, such as papillary thyroid cancer (PTC), follicular thyroid cancer (FTC), and other malignancies, showed similar yearly occurrence in both study periods (PTC: 5 vs. $10 \mathrm{pat} / \mathrm{y}$ in each period $[P=0.01]$; FTC: 6 vs. 5 pat $/ \mathrm{y}$ per period $[P=0.04]$; others: 4 vs. 4 pat/y per period $[P=\mathrm{NS}])$. The indications for surgery were the same in both study periods, namely, growing thyroid nodules, suspected malignancy, mechanical disturbances, and in a few cases cosmetic reasons (asymmetry, size of the tumor).

As is usual in a teaching hospital, various surgeons performed the operations during the study periods. A staff surgeon supervised the operations in all cases. Intraoperative frozen sections were taken and histological analyses of thyroid tissue were performed in every patient and directly influenced the extent of surgery.

During the first study period (1972-1990), following Kocher's recommendation, mainly subtotal thyroid resections (leaving a remnant of thyroid tissue of $>8 \mathrm{ml}$ ) or merely enucleations of thyroid nodules (in order to avoid dependence on thyroid hormone substitution) were performed (80\%; Fig. 1). Less than $20 \%$ of the operations before 1990 involved complete lobectomies (hemithyroidectomies).

From 1991 onward, the surgical approach was more extensive: in patients with strictly unilateral thyroid dis- ease a lobectomy was performed on the affected side. Most of the patients, however, presented with nodules in both thyroid lobes, where a bilateral lobectomy was performed, unless macroscopically normal thyroid tissue could be left in place on one side, in which case a subtotal resection (leaving $2-4 \mathrm{ml}$ of thyroid tissue) was performed on one side and a complete lobectomy on the contralateral side (Fig. 1).

Defined surgical technique was performed in both study periods without modifications. ${ }^{13-16}$ A staff surgeon was present in all operations in both study periods. In contrast to the first study period (1972-1990), the recurrent laryngeal nerves and the parathyroid glands were obligatorily visualized during the second period, from 1991 to 2002. In the case of accidental devascularization of the parathyroid gland the parathyroid was transplanted in the sternocleidomastoid muscle. Permanent recurrent laryngeal nerve palsy rates (defined as persistence of palsy for more than 6 months) and rates of permanent hypoparathyroidism (defined as persistence of hypocalcemia for more than 6 months) were analyzed. Indications for reoperative thyroid gland surgery were mechanical disturbances, compression of the trachea, rapid thyroid growth, or suspicion of malignancy. ANOVA was used to analyze linear regression models, and post-tests were done with the KruskalWallis method. Hypothesis testing was performed with Fisher's exact test using Number Cruncher Software (NCSS, Kaysville, UT, USA). Significance levels were set at a $P$ value of $\leq 0.05$. 


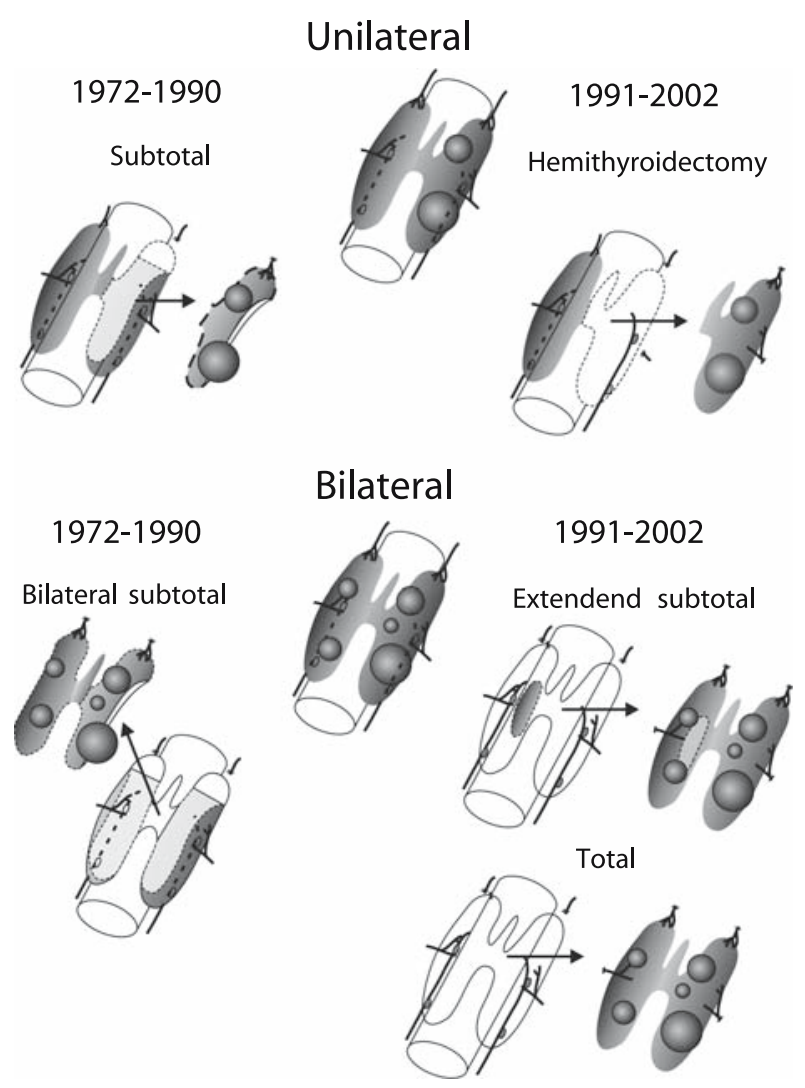

Figure 1. Schematic drawing of the amount of thyroid tissue resected before and after 1991. In unilateral disease (top), a subtotal thyroid resection that left significant tissue behind (> $8 \mathrm{ml}$ ) was performed in the first study period (1970-1990), whereas a hemithyroidectomy (lobectomy) with resection of the isthmus and the lobus pyramidalis was performed in the second study period and the laryngeal nerve (dotted line) as well as both parathyroid glands were exposed (1991-2002). Bi-lobar disease (bottom) was treated with bilateral subtotal thyroidectomy or cyst enucleation before 1991 and with hemithyroidectomy plus contralateral lobectomy or an extended subtotal resection (neartotal resection)-if macroscopically normal tissue could be left in place-after 1991.

\section{RESULTS}

\section{Injury of the Inferior Laryngeal Nerve (Recurrent Laryngeal Nerve)}

Analyzing the 2,867 thyroid interventions with 3,488 nerves at risk in the first study period, from 1972 to 1990 , we noticed that the recurrent laryngeal nerve palsy rate was $3.6 \%$ (105 out of 2,925) for primary operations and $5.3 \%$ (30 out of 563 ) for interventions treating recurrent disease (Table 3). Comparing these data with the results from the second period, 1991-2002 (with obligatory visualization of the recurrent laryngeal nerve and the parathyroid glands), which included 1,482 thyroid opera- tions with 2,297 nerves at risk, we found a significant decrease in the recurrent laryngeal nerve palsy rate to $0.9 \%$ (20 out of 2,128; $P<0.001$; Table 3 ). This reduction in nerve lesions corresponds to a relative risk reduction (RRR) of $74 \%$ and an absolute risk reduction (ARR) of $2.66 \%$. Hence, every 38th patient would profit from the more extended operation (number needed to treat $[N N T]=38$ patients). The laryngeal nerve palsy rate after surgery for thyroid disease recurrence decreased non-significantly from $5.3 \%$ to $4.7 \%$ (8 out of 169) from the first to the second observation period.

To evaluate if differences between the 2 observation groups (1972-1990 and 1991-2002) arose from an unknown effect over time, we performed a subgroup analysis of the variation of the rate of laryngeal nerve palsy within the observation time. Figure 2 shows the box-plot of laryngeal nerve injuries in 5 (6)-year intervals, confirming that changes did indeed occur between 1990 and 1991 rather than over the period before or after the change of technique. A time effect would be noticeable by a tendency of the intervals toward lower palsy rates over time. Post-test analysis with the Kruskal-Wallis test showed a significant difference between the intervals of the first observation period and the second period $(P=0.005)$.

\section{Hypoparathyroidism}

The incidence of permanent hypoparathyroidism ( $>1$ year) at the initial operations was $3.2 \%$ (93 out of 2,867 ) for the first study period and $0.6 \%$ (9 out of 1,482 ) for the second $(P<0.01$; Table 3$)$. Hence, our data show, somewhat unexpectedly, that postoperative permanent hypoparathyroidism was significantly less frequent after the more extended operation (with obligatory visualization of the parathyroid glands). The relative risk reduction (RRR) was $81 \%$, corresponding to an ARR of $2.6 \%$, which translates into an NNT of 38 , so that again, in every 38th patient hypoparathyroidism could be avoided by the radical approach.

\section{Incidence of Reoperation for Disease Recurrence}

The number of patients who were referred to our institution for reoperation due to thyroid disease recurrence declined over the study periods (Fig. 3A). The incidence of reoperations decreased from $11.1 \%$ (319 out of 2,867 ) in the first study period to $8.5 \%$ (126 out of 1,482 ) in the second period $(P=0.007)$. Analysis of recurrent disease rates over both observation periods by linear regression 
Table 3.

Morbidity of thyroid surgery

\begin{tabular}{|c|c|c|c|c|c|}
\hline & \multicolumn{2}{|c|}{$1972-1990$} & \multicolumn{2}{|c|}{ 1991-2002 } & Relative risk (Cl 95\% LL-UL) \\
\hline Thyroid operations & & & & & \\
\hline Nerves at risk & & & & & \\
\hline First resection & & & & & \\
\hline Resection for recurrence & & & & & \\
\hline \multicolumn{6}{|l|}{ Recurrent nerve palsy } \\
\hline Primary surgery & 105 & $3.6 \%$ & 20 & $0.9 \%{ }^{*}$ & $0.26(0.16-0.43)$ \\
\hline Recurrent surgery & 30 & $5.3 \%$ & 8 & $4.7 \%$ & \\
\hline Hypoparathyroidism & 93 & $3.2 \%$ & 9 & $0.6 \%{ }^{*}$ & $0.19(0.09-0.38)$ \\
\hline
\end{tabular}

95\% Cl: 95\% confidence interval, LL: lower limit, UL: upper limit (of 95\% confidence interval).

${ }^{\star} P<0.001$

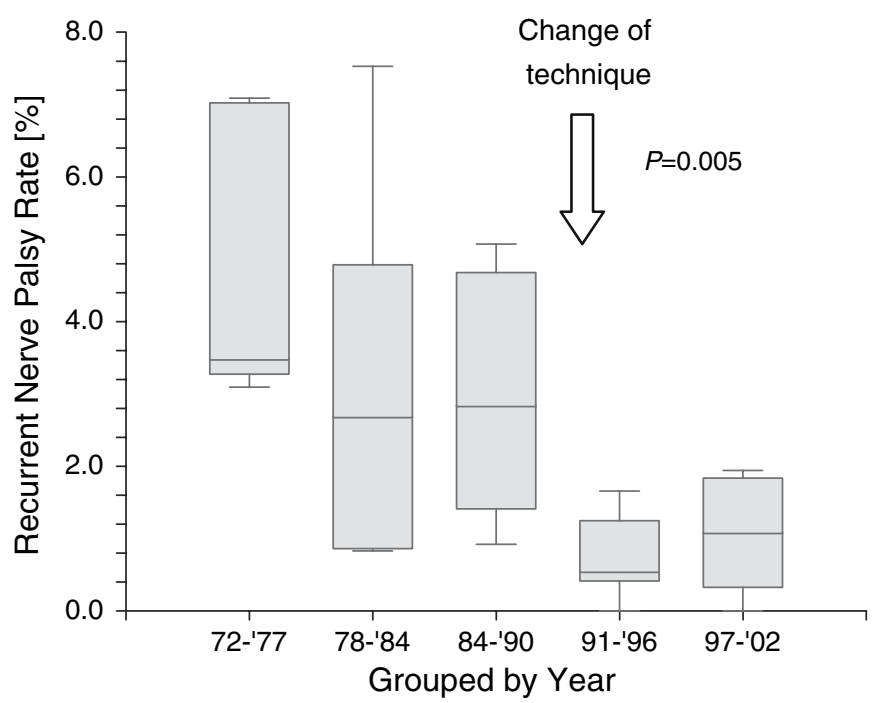

Figure 2. Subgroup analysis for laryngeal nerve palsy rate over the years. Nerve lesions remained at similar levels within the observed groups (1972-1990/1991-2002). The box-plot diagram depicts the rate of nerve lesions during each 5 (6)-year interval. The horizontal lines within the box represent the median, and boxes give the interquartile range (IQR) of $50 \%$ (upper: $75 \%$, lower: $25 \%$ ). T-bars are given for the $75 \%$ plus 1.5 times the IQR and $25 \%$ minus 1.5 times the IQR respectively. Whereas groups within the observation periods (1972-1990 and 1991-2002) did not differ significantly from each other, groups from the first period had a significantly higher incidence of nerve lesions after primary surgery than the 2 groups treated with the total hemithyroidectomy approach $(P=0.005$; Kruskal-Wallis test).

showed that the incidence remained stable over the first study period (1972-1990; $P=0.73$ ), whereas a trend toward a decreased incidence was found in the second study period ( $P=0.09$; Figs. 2C, 3B). Because follow-up cannot be guaranteed to be complete, we performed a case-cohort analysis with a calculated odds ratio (OR) of 0.74 (95\% Cl: 0.59-0.92), which showed a 1.3 times higher risk for patients from the earlier study period (19721990) who needed reoperation.

\section{DISCUSSION}

\section{Morbidity of Extensive Surgery}

Concern that a more extensive surgical approach to (nodular) goiter would increase peri- and postoperative morbidity has been raised by endocrinologists and surgeons alike, especially in countries where medico-legal issues are of concern. In this prospective cohort study we found that extensive surgical thyroid resections lead to a significant reduction in surgical morbidity compared with limited (subtotal) resection: in our study populations the recurrent laryngeal nerve palsy rate dropped 4-fold from $3.6 \%$ in the subtotal hemi-thyroidectomy group to $0.9 \%$ in the complete hemi-thyroidectomy group. These findings correlate with the data of a large meta-analysis on recurrent laryngeal nerve palsy associated with thyroid surgery. The meta-analysis compared visualization of the recurrent laryngeal nerve (which is necessary in total thyroidectomy) to "blind," subtotal thyroid resections without visualization of the recurrent laryngeal nerve during thyroid gland surgery. It showed a significant reduction in palsy rates from $6.1 \%$ in the "blind" group to $2.5 \%$ in the "visualized" group. ${ }^{17-19}$ Interestingly, the percentage decrease in the rate of nerve lesions at our institution $(75 \%)$ was even higher than the rate in the meta-analysis (59\%). Routine intraoperative visualization of the laryngeal nerve and the parathyroid glands (as performed in the second study period) as well as continually improving anatomical knowledge of the performing surgeon, probably both, contributed to this reduction in postoperative laryngeal nerve palsy in extensive goiter 

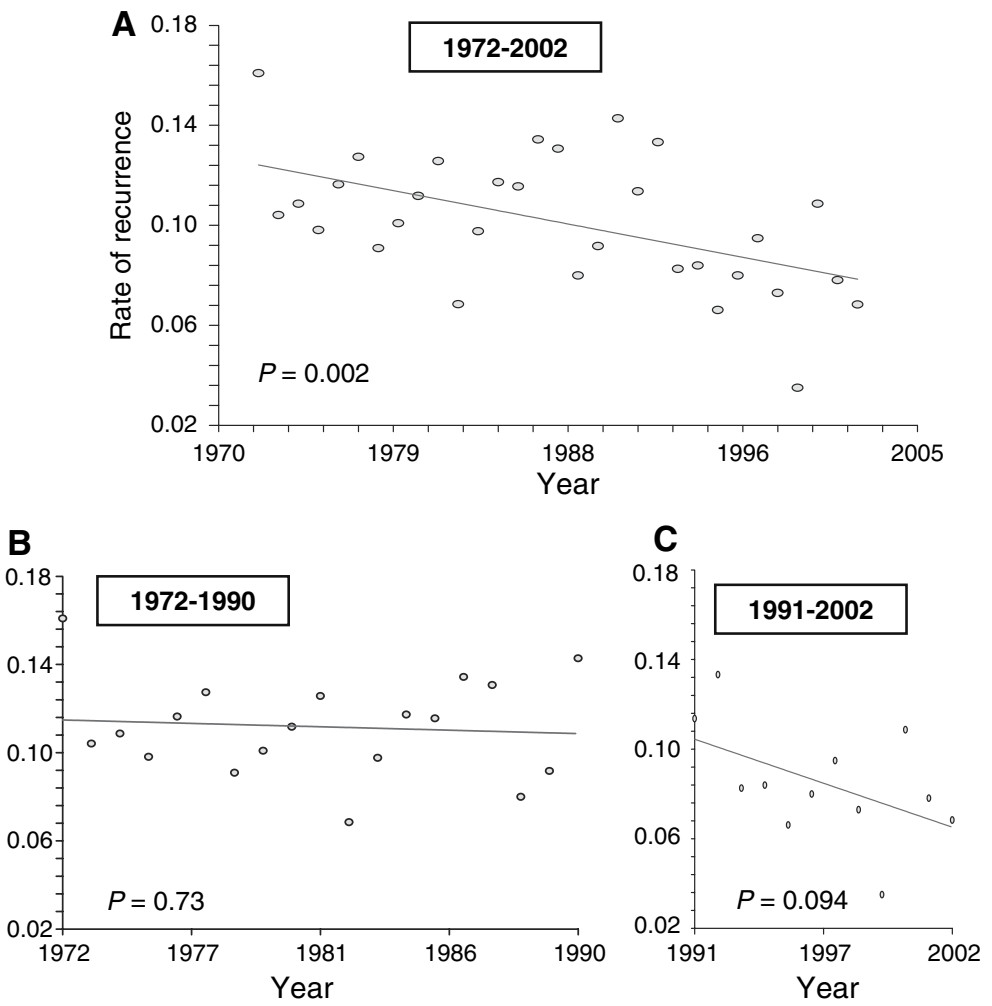

Figure 3. Rates of operations for recurrent thyroid disease over time. A. Rate of patients operated on for recurrent thyroid disease per year. Dot-plot with linear regression line of the rate of recurrent thyroid operations each year. Dots mark the rates and the line represents the linear regression model. $P$ values are calculated by one-way ANOVA. During the whole observation period the incidence for reoperations declined significantly. B. Rate of reoperation for recurrent disease during the first observation period (1972-1990). Linear regression shows little difference in the rate of reoperations over time. C. Rate of reoperations for recurrent disease during the second observation period (1991-2002). Despite the significant decrease in reoperation rates (from 11.1\% to $8.5 \%)$ between the 2 study periods $(P=0.007)$, the slope of the linear regression model shows no significance, but a tendency toward decreased rates of reoperation is evident. The slope served to verify the continuing decrease in reoperations in the second period, which will, likely, reach significance in time.

surgery. $^{20-23}$ This perfection of surgery could also be reflected in the 5-fold drop in the postoperative hypoparathyroidism rate from $3.2 \%$ to $0.6 \%(P<0.001)$.

An additional advantage of a more extensive surgical strategy is that expensive, time-consuming preoperative imaging studies-scintigraphy, MRI, CT scans (contraindicated when postoperative radio-iodine-therapy is considered)-become redundant. In most cases preoperative sonography will be sufficient.

It is of interest to note that other pioneers of thyroid surgery were already advocating the complete lobectomy with visualization of the recurrent laryngeal nerve as the gold standard in the 1970s. However, this complete resection was performed in only a few centers, mainly in the USA, and it was not considered "standard therapy" for benign thyroid disease in most centers due to lack of reliable data. However, nowadays, surgeons in the USA specialized in endocrine surgery increasingly prefer the more extensive approach as described in this study. Especially in regions where goiter disease is endemic, the historically grown approach of limited resection is still quite widespread.

\section{Incidence of Disease Recurrence}

Despite the elimination of iodine deficiency the incidence of goiter remained remarkably high in Switzerland. The astonishingly high incidence of recurrent goiter necessitating secondary thyroid surgical treatment, which is associated with significant morbidity was particularly bothersome. Conservative treatment with thyroid hormones to prevent goiter recurrence after subtotal thyroidectomy showed only limited efficacy. ${ }^{24-29}$ The endocrinology research department of the University of Bern has recently described the molecular biological phenomenon that epithelial cells of the thyroid could alter their phenotype during a patient's life. These changes in the phenotype are followed by clonal growth leading to functional as well as morphologic polymorphism that has the potential to occur anywhere in the 
whole thyroid tissue. The episodic replication of these multiple clusters (nodular growth) seems to be dependent on the over-expression of growth factors $(\mathrm{JgF}, \mathrm{EgF}$, RAS). ${ }^{24,30-32}$ Thus, it is tempting to speculate that reducing the amount of cells that have the potential of clonal morphological change surgically would reduce the incidence of disease recurrence. The findings of the present study confirm that a more extensive surgical approach can reduce the incidence of recurrent goiter significantly (from $11.1 \%$ to $8.5 \% ; P=0.007$ ). ${ }^{13}$ This more extensive approach includes the total resection of all nodular thyroid tissue with at least a lobectomy (hemithyroidectomy) on one side and, if indicated by the existence of bilateral nodular disease, a total or subtotal lobectomy on the contralateral side. However, we have to acknowledge that the duration of the observational period (12 years) is rather short, because disease may recur even after 20 years.

\section{Surgery for Recurrent Disease}

The newly gained molecular knowledge in the pathogenesis of goiter could explain why $78 \%$ of the recurrences in this study were seen in euthyroid benign goiters. Reoperations for recurrent thyroid disease harbor the potential for significant morbidity. ${ }^{13,18,19}$ That is why redo surgery is often delayed until the clinical situation has worsened so much that a second surgical intervention is inevitable. The possibility of reducing the chance of goiter recurrence by doing an extended thyroid resection at the initial operation, as described in this study, thus offers an obvious advantage. This approach also lessens the risk of laryngeal nerve lesions at a subsequent operation for recurrent disease because only one side and often unresected (untouched) side will have to be explored. Hence, complete thyroidectomy on the diseased side will not only directly reduce morbidity at the first operation, but it will also reduce the incidence of recurrent disease and its risky re-explorations. In addition, only one side will be exposed to potential nerve and parathyroid damage if a reoperation should be necessary. Thus, lobectomy instead of subtotal thyroidectomy at the initial operation reduces the risk of significant morbidity at 3 different levels. As mentioned in the previous paragraph, given the not insignificant incidence of recurrent goiter disease, patients with any nodular disease on the contralateral side should be encouraged to proceed with near-total thyroidectomy or more complete resection.

\section{Drawback of the More Extensive Surgical Approach}

The more extensive resection of thyroid tissue compared with subtotal thyroidectomy or enucleation alone, would be expected to increase in the requirement for hormonal substitution therapy.

Preliminary analysis of our data show that postoperative substitution with thyroid hormones is, indeed, prescribed in $50 \%-75 \%$ of patients treated with the more extensive approach. However, it is extremely difficult to assess whether substitution is indicated for all these patients because postoperative management of patients operated for goiter remains in the hands of general practitioners. Unfortunately, many still believe that goiter recurrences can be prevented by thyroid hormone treatment, which they therefore prescribe to all patients who have undergone goiter surgery, independent of their thyroid metabolic state.

\section{Statistical Considerations}

In this cohort study we prospectively analyzed 2 populations treated for thyroid disease for morbidity associated with the intervention. A major concern of non-concurrent studies, in general, is the influence of time-dependent factors not accounted for. Thus, we evaluated the 2 groups for differences in additional factors that could influence the outcome (Table 2). Because during both observation periods the frequency and indications for thyroid surgery remained identical, we assumed that no changes in referral patterns or discrepancies in the rate of disease entities occurred. Furthermore, analysis of time-dependent factors showed a constant rate of laryngeal nerve lesions within the 2 observation periods (1972-1990 and 1991-2002; Fig. 3), further indicating that the strongly significant differences between the two groups are mainly due to the change in surgical technique rather than to an unknown effect over time. Ninety-nine percent of the patients were prospectively followed for postoperative recurrent laryngeal nerve lesions and hypoparathyroidism. However, only patients referred to our clinic were included in the analysis of goiter recurrence. The probability that patients with recurrent disease were lost to follow-up because they were referred to clinics other than the single specialized referral center for thyroid disease in the region is quite small. Considering the low probability of committing a type I error, we therefore suggest that the observed difference in recurrence rates is due to the change in the treatment strategy. 


\section{CONCLUSION}

The data presented in this study support the recommendation to perform at least a 1-sided lobectomy combined with either total or subtotal thyroidectomy of the contralateral side if indicated at the initial operation for benign thyroid disease. Hence, Theodor Kocher's "Gold standard" (subtotal resections or enucleations without perioperative visualization of the recurrent laryngeal nerve or the parathyroids) would be no longer advisable.

The more extensive approach (with visualization of the recurrent laryngeal nerve and the parathyroid glands) is based on the newly gained knowledge of goitrogenesis as a molecular biological and genetic disease. It not only has the advantage of minimizing the remaining thyroid tissue and, hence, reducing the number of clones that have the potential to recur, but also to significantly reduce the rate of recurrent laryngeal nerve lesions as well as to avoid postoperative hypoparathyroidism. Hence, a reduction in overall morbidity of the surgical treatment of goiter can be seen:

1. At the level of reduced morbidity of the initial operation

2. At the reduction in goiter recurrence

3. At the level of decreased morbidity in the case of recurrent thyroid disease: if reoperation is required the risk of potential bilateral nerve damage is avoided, as the resection will be limited to 1 side only

However, our data do not allow postoperative hypothyroidism to be analyzed, requiring hormone substitution, due to the more extensive approach advocated here.

Interestingly, the extensive surgical resection to benign thyroid disease was the initial intention of Theodor Kocher when he conducted his first study involving over 100 thyroidectomies in 1883. At that time untreatable postoperative hypothyroidism and hypoparathyroidism combined with anesthesiologic difficulties forced him to advocate his "criterion standard" of subtotal resection that would stand for a century. ${ }^{1}$ Today, however, based on the knowledge gained in goitrogenesis and based on the significantly reduced morbidity shown in this study, the time has come to advocate his initial intention of extensive resection as the "gold standard" in the surgical treatment of benign thyroid disease.

\section{ACKNOWLEDGEMENTS}

We would like to thank Prof. R. Berchtold, M.D.; Prof. L.H. Blumgart, M.D.; Prof. M.W. Büchler, M.D. former chairmen of the Department of Visceral and Transplantation Surgery at the University of Bern for their continuous support. We would especially like to thank H.E. Wagner, M.D. for his personal contribution and J. Wurz for the proofreading of the manuscript.

\section{REFERENCES}

1. Kocher T. Über die Kropfexstirpation und ihre Folgen: Archiv für Klinische Chirurgie; 1883.

2. Bornhauser S. (1951) Zur Geschichte der Schilddrüsenund Kropfforschung im 19. Jahrhundert1 Sauerländer, Aarau.

3. McGreevy PS, Miller FA. Biography of Theodor Kocher. Surgery 1969;65:990-999.

4. Troehler U. (1984) Der Nobelpreisträger Theodor Kocher 1841-19171 Birkhäuser, Basel.

5. Welbourn RB, Friesen SR, Johnston IDA, et al. (1990) The history of endocrine surgery Praeger, Westport.

6. Boeni T, Bonjour E, Boschung U, et al. (1991) Theodor Kocher, 1841-1917 Huber, Bern.

7. Roeher $\mathrm{H}$. Theodor Kocher Lecture. 22nd Annual Meeting of Working Group for Endocrine Surgery (CAEK) of the German Surgical Society, Frankfurt; 2003.

8. Lauener P. Ein Beitrag zur Begründung der Notwendigkeit einer planmässigen Kropfbekämpfung. Schweiz Med Wochenschr 1936;66:189.

9. Lauener P. 15 Jahre Kropfbekämpfung in den Schulen der Stadt Bern. Schweiz Med Wochenschr 1936;66:207.

10. Lauener P. Statistische Erhebungen über den Kropf bei Schulkindern im Kanton Bern vor und nach Einführung des iodierten Kochsalzes. Schweiz Med Wochenschr 1939; 69:455.

11. Lauener P. Kropf und Kropfbekämpfung in der Schweiz. Z Arztl Fortbild 1941;38:179.

12. Burgi $H$, Supersaxo Z, Selz B. lodine deficiency diseases in Switzerland one hundred years after Theodor Kocher's survey: a historical review with some new goitre prevalence data. Acta Endocrinol (Copenh) 1990;123:577-590.

13. Seiler CA, Glaser $C$, Wagner HE. Thyroid gland surgery in an endemic region. World J Surg 1996;20:593-596discussion 596-597.

14. Reeve TS, Delbridge L, Cohen A, et al. Total thyroidectomy. The preferred option for multinodular goiter. Ann Surg 1987;206:782-786.

15. Jatzko GR, Lisborg PH, Muller MG, et al. Recurrent nerve palsy after thyroid operations-principal nerve identification and a literature review. Surgery 1994;115:139-44.

16. Seiler CA, Schafer M, Buchler MW. Surgery of the goiter. Ther Umsch 1999;56:380-384.

17. Schilling MK, Seiler $C$, Schafer $M$, et al. Prevention of $N$. recurrens paresis after thyroidectomy-a meta-analysis. Ther Umsch 1999;56:396-399. 
18. Dralle $\mathrm{H}$, Pichlamayr R. Decrease of risks in reoperation for benign struma. Chirurg 1991;62:169-175.

19. Levin KE, Clark $A H$, Duh $Q Y$, et al. Reoperative thyroid surgery. Surgery 1992;111:604-609.

20. Gemsenjager E. Surgical technique in interventions on the thyroid gland. Chirurg 1993;64:725-731.

21. Seiler CA, Wagner HE. The non-recurrent inferior laryngeal nerve. An important rarity in thyroid gland surgery. Chirurg 1994;65:358-360.

22. Seiler CA, Wagner HE. Recovery from bilateral vocal cord paralysis following thyroid surgery. $\mathrm{Br} \mathrm{J}$ Surg 1994; 81:1760.

23. Wagner HE, Seiler C. Recurrent laryngeal nerve palsy after thyroid gland surgery. Br J Surg 1994;81:226-228.

24. Teuscher J, Peter HJ, Gerber H, et al. Pathogenesis of nodular goiter and its implications for surgical management. Surgery 1988;103:87-93.

25. Westermark K, Persson CP, Johansson $\mathrm{H}$, et al. Nodular goiter: effects of surgery and thyroxine medication. World $\mathrm{J}$ Surg 1986;10:481-487.

26. Pickardt CR, Scriba PC. What does the internist expect of surgeons in surgery of benign thyroid diseases?. Chirurg 1991;62:157-61.
27. Hegedus L, Nygaard B, Hansen JM. Is routine thyroxine treatment to hinder postoperative recurrence of nontoxic goiter justified?. J Clin Endocrinol Metab 1999;84:756760.

28. Rojdmark J, Jarhult J. High long term recurrence rate after subtotal thyroidectomy for nodular goitre. Eur J Surg 1995;161:725-727.

29. Persson $\mathrm{CP}$, Johansson $\mathrm{H}$, Westermark $\mathrm{K}$, et al. Nodular goiter-is thyroxine medication of any value?. World J Surg 1982;6:391-396.

30. Aeschimann S, Kopp PA, Kimura ET, et al. Morphological and functional polymorphism within clonal thyroid nodules. J Clin Endocrinol Metab 1993;77:846-851.

31. Studer H, Gerber H, Zbaeren J, et al. Histomorphological and immunohistochemical evidence that human nodular goiters grow by episodic replication of multiple clusters of thyroid follicular cells. J Clin Endocrinol Metab 1992; 75:1151-1158.

32. Studer H, Peter HJ, Gerber H. Natural heterogeneity of thyroid cells: the basis for understanding thyroid function and nodular goiter growth. Endocr Rev 1989;10:125-135. 